\title{
Bladder Carcinoma after ABVD Chemotherapy for Hodgkin's Lymphoma: A Case Report
}

\author{
Aparna Gangopadhyay Partha Nath \\ Mofizur Rahman Khan Jaydip Biswas \\ Chittaranjan National Cancer Institute, Kolkata, India
}

\section{Key Words}

Hodgkin's lymphoma · ABVD · Chemotherapy · Bladder carcinoma

\begin{abstract}
The treatment of lymphomas may result in the development of second malignancies, as evident by the numerous reports in the literature. Treatment with cyclophosphamide-based chemotherapy regimens may lead to bladder lesions such as haemorrhagic cystitis and also to carcinoma of the urinary bladder. Previous pelvic radiotherapy treatment is also implicated as a cause for local second cancers. We present the case of a patient treated for Hodgkin's lymphoma, who was diagnosed with transitional cell carcinoma of the bladder soon after treatment completion. On completion of 6 cycles of ABVD chemotherapy the patient was on follow-up. Two months after treatment completion the patient complained of dysuria and was investigated for a suspected urinary tract infection. Urine microscopy did not reveal any abnormality. Symptomatic treatment was prescribed and cystoscopy was arranged. The cystoscopic findings suggested an irregular growth overlying the trigone and the biopsy reported it as transitional cell carcinoma. This case report demonstrates that symptoms attributed to common medical causes in patients treated for cancer may be a sign of second malignancy. This case report also demonstrates the need for a thorough evaluation of patients' complaints during follow-up, although the likelihood for the occurrence of a second malignancy may be low. The assumption that these symptoms were due to a commonly occurring urinary tract infection would have had serious implications leading to a delay in the treatment of the bladder cancer.
\end{abstract}

\section{Introduction}

Cyclophosphamide-based chemotherapy is a well-known agent triggering second malignancies in many patients. Similarly, radiation-induced cancers, mainly sarcomas are commonly seen at sites that have been included in radiation fields. 
Studies have shown a definite association between treatment for lymphoma and development of bladder carcinoma [1]. In some cases this may occur in the backdrop of cyclophosphamide-induced cystitis. Uroprotection with Mesna administered during chemotherapy has been thought to provide some protection against cystitis in these patients.

Usually, treatment of patients with various alkylating agents induces cancer of many different types, which includes that of the kidney and bladder. Statistically, the most common secondary malignancies that result from treatment of lymphomas are leukemias [2] and many solid tumours, especially cancers of the lung, breast, thyroid, bone, soft tissue, stomach, oesophagus, colon and rectum, uterine cervix, head and neck, and mesothelioma $[3,4]$. These tumours occur primarily after radiation therapy or with combined modality treatment, and approximately $75 \%$ occur within radiation ports. At a 15-year follow-up, the risk of second solid tumours approximates $13 \%$ as per estimates in the literature $[3,5]$.

Occurrence of bladder cancers has not been commonly documented in patients treated with regimens that exclude cyclophosphamide or ifosfamide. Moreover, a search of PubMed does not reveal any reports on the incidence of developing bladder cancer within a few weeks of completion of chemotherapy in lymphoma patients.

We present the case of a patient, diagnosed with Hodgkin's lymphoma, who developed bladder carcinoma presenting with features of urinary tract infection within 8 weeks of completion of chemotherapy. After treatment with transurethral resection of the bladder tumour and intravesical immunotherapy, a favourable response to treatment was obtained. The patient is presently on follow-up.

\section{Case Presentation}

Our patient is a 35-year-old man. The patient was in his usual state of health until February 2010 when he developed multiple swellings on both sides of the neck. Apart from the neck swellings he had no other significant complaints, and no loss of weight or fever. Examination revealed multiple firm mobile nodes measuring a maximum of $2 \mathrm{~cm}$ in diameter involving levels II, III and IV on both sides of the neck with no associated tenderness. No other peripheral nodes were palpable.

The patient underwent a fine needle aspiration of a neck swelling at an outside hospital, which revealed features suggestive of a lymphoproliferative disorder. He underwent an excision biopsy of a node at our institute. The histology was reported as classical Hodgkin's lymphoma, mixed cellularity type (fig. 1). Systemic workup did not reveal any evidence of disease beyond the neck. He was initiated on chemotherapy with ABVD regimen. The response to chemotherapy was favourable and he completed 6 cycles of chemotherapy. Post-treatment investigations showed evidence of complete response. He was accordingly asked to attend our institute for regular follow-up. During one of his follow-up visits on January 5, 2011, he complained of dysuria. Symptomatic medications were prescribed and a urine microscopy and Gram stain was requested prior to initiation of antibiotics. The patient reported back in a week with no relief of symptoms. No abnormality was found in the microscopy and Gram stain report. Cystoscopic evaluation was requested this time. On January 28, 2011, he underwent cystoscopy. An irregular growth on the bladder mucosa measuring around $1.5 \mathrm{~cm}$ was noted overlying the trigone and a transurethral resection of the bladder tumour was arranged. The entire lesion was resected at its base and fulgurated. No other abnormality was noted on inspection of the other areas of bladder mucosa. The histology ( $\underline{\text { fig. } 2}$ ) revealed transitional cell carcinoma that had invaded the lamina propria. Muscle invasion was not reported.

Treatment options for further management of his bladder tumour were discussed. It was agreed that he would receive intravesical BCG. He started his first dose of intravesical treatment (120 mg) on 
February 10, 2011. He tolerated the 6 doses of weekly intravesical BCG well, with occurrence of mild cystitis at week 5, which did not require treatment interruption. A cystoscopy done on completion of the 6 doses on March 30, 2011 showed no recurrence or any new lesion elsewhere on the mucosa. It was agreed with the patient that he would proceed with maintenance therapy at three-monthly intervals for 1 year. Check cystoscopies at three-monthly intervals were performed. His cystoscopy done on January 24, 2012 did not show any evidence of disease. He is now on follow-up.

\section{Discussion}

Second malignancies represent an important iatrogenic complication of the treatment of neoplasms. Hodgkin's disease has been treated mainly with two chemotherapy schedules, MOPP (nitrogen mustard, Oncovin, procarbazine and prednisone), which includes alkylating agents, and ABVD (Adriamycin, bleomycin, vinblastine and dacarbazine), which includes topoisomerase II inhibitors. Due to the types of agents used, patients with Hodgkin's disease often develop secondary leukemias. As noted by Salas et al. [6], specific cytogenetic changes are associated with the therapy-related myelodysplastic syndrome or therapy-related acute myeloid leukemia that occurs as a sequela of their chemotherapy. Interestingly, they noted that at the cytogenetic level, patients treated with alkylating agents and with topoisomerase II inhibitors show distinct genetic alterations. These findings potentially have a bearing on the prognostication and treatment of these haematological malignancies.

Among patients of Hodgkin's lymphoma, follow-up data in long-term survivors suggest a significant risk of second solid tumours as well, with the risk of certain cancers remaining significantly high in these patients. Data analysed by Dores et al. [4] from population-based cancer registries across North America and Europe revealed significantly elevated risks of stomach, female breast, and uterine cervix cancers up to 25 years after treatment completion in Hodgkin's disease survivors.

Várady et al. [7] reported that fewer than 5\% of 665 patients treated for Hodgkin's lymphoma at their institute from 1978 to 1996 developed second malignancies. These patients had received either chemotherapy alone or both chemotherapy and radiotherapy. Haematological malignancy occurred after a mean interval of 6.1 years from treatment completion. This was attributed to the alkylating agents they had received for treatment of Hodgkin's lymphoma.

Solid tumours that occurred included mostly carcinomas, but also sarcomas and melanoma. Quite a few of these were found in previously irradiated regions. The mean latency period to developing the second cancer in patients developing solid tumours was 8.3 years.

Maurizi et al. [8] attempted to examine the occurrence of solid tumours in relation to the different types of therapy (radiotherapy, chemotherapy and radiochemotherapy; splenectomy or splenic irradiation versus no splenectomy-no splenic irradiation) received by patients treated for Hodgkin's disease. The average follow-up period was 72 months. Their patients had received ABVD or MOPP/ABVD hybrid chemotherapy regimens as sole or part of their treatment. According to multivariate analysis, their findings supported that increasing age, specifically $>40$ years, at the time of diagnosis of Hodgkin's lymphoma is the most important factor in the risk of developing solid 
tumours. They also noted that treatment of Hodgkin's lymphoma with chemotherapy alone increases the risk of second solid tumours.

Bonadonna et al. [9] reported their experience regarding survival in 811 patients with intermediate and advanced Hodgkin's lymphoma treated at the Milan Cancer Institute. They noted that ABVD chemotherapy was more efficacious than the MOPP regimen in significantly reducing the risk of lymphoma progression and death. However, they noted that a significant risk of developing second cancers also remained, especially in those receiving extensive irradiation. In their group the total risk of developing a second malignancy was $22.2 \%$.

The analysis by Valagussa et al. [10], with a median 43 months of follow-up revealed that solid tumours occurred only in patients given radiotherapy with or without adjuvant chemotherapy, and acute non-lymphoblastic leukemia occurred only after treatment with MOPP or modified MOPP regimens. Neither solid tumours nor acute non-lymphoblastic leukemia occurred in patients given ABVD.

Valagussa et al. [11] reported on a retrospective analysis of 1,032 patients with Hodgkin's disease admitted to their Institute between 1965 and 1978 and treated with radiotherapy or chemotherapy or both modalities. The analysis revealed that within 10 years from initial therapy, no patient receiving ABVD developed second cancers, whether they received radiotherapy or not. This prompted them to report that there was an absence of treatment-induced second neoplasms after ABVD chemotherapy in Hodgkin's disease.

As survival rates in Hodgkin's lymphoma have improved over the years the side effects related to treatment are a matter of concern in these patients, especially the occurrence of second cancers. With advances in treatment, there has been significant modification of chemotherapy agents, shifting to safer drugs that reduce not only the chance of second malignancies but also other side effects including fertility issues. As noted in the literature, the ABVD chemotherapy widely in use is safer and has done away with some of the issues associated with MOPP or MOPP hybrid chemotherapy. More efficient chemotherapy has made magna field irradiation a thing of the past in Hodgkin's lymphoma treatment. There has also been a paradigm shift in radiotherapy delivery techniques, which have greatly reduced volumes of tissue receiving the prescription dose. Safer treatment has transformed survival and side effects in these patients. Consequently, surveillance for second cancers is of great importance in this group.

Numerous reports in the literature suggest that cyclophosphamide-treated patients are at a greater risk of developing bladder carcinoma, and the risk may be 4- to 10-fold higher than in the general population $[12,13]$. The use of cisplatin and delivery of radiotherapy to the pelvis have also been implicated as risk factors for future development of bladder carcinoma [14]. Despite the extensive number of reports on the occurrence of bladder carcinoma due to different chemotherapy regimens with or without radiation therapy, no reports linking bladder cancers to ABVD chemotherapy have been noted on a comprehensive search of the literature.

The occurrence of bladder carcinoma after ABVD chemotherapy and that too within a short period of treatment completion is very rare. Our experience emphasizes the need for a higher degree of suspicion regarding the development of second cancers 
during follow-up of patients treated for lymphoma. With improved survival, early detection and treatment of solid tumours that may arise as a consequence of treatment of Hodgkin's lymphoma is of immense importance. It is essential that at each follow-up visit any new symptoms are carefully followed up and investigated in these patients. Not all patients presenting with second cancers will have received the chemotherapy agents reportedly linked to that particular tumour type.

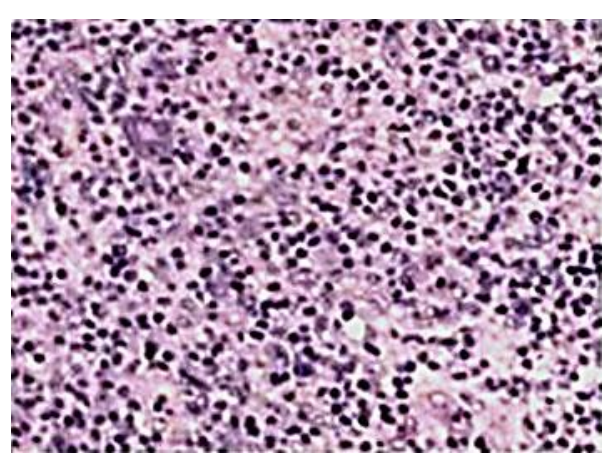

Fig. 1. Histology of excised node showing mixed cellularity Hodgkin's lymphoma.

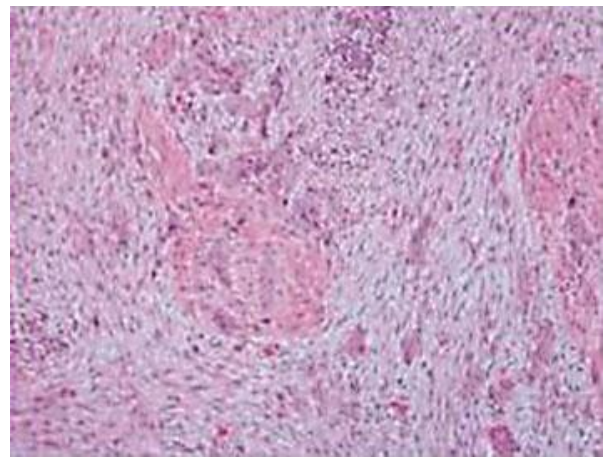

Fig. 2. Histology of bladder growth removed by transurethral resection. 


\section{References}

1 Pathak AB, Advani SH, Gopal R, Nadkarni KS, Saikia TK: Urinary bladder cancer following cyclophosphamide therapy for Hodgkin's disease. Leuk Lymphoma 1992;8:503-504.

-2 Valagussa P, Santoro A, Fossati-Bellani F, Banfi A, Bonadonna G: Second acute leukemia and other malignancies following treatment for Hodgkin's disease. J Clin Oncol 1986;4:830-837.

-3 Hancock SL, Hoppe RT: Long-term complications of treatment and causes of mortality after Hodgkin's disease. Semin Radiat Oncol 1996;6:225-242.

-4 Dores GM, Metayer C, Curtis RE, Lynch CF, Clarke EA, Glimelius B, Storm H, Pukkala E, van Leeuwen FE, Holowaty EJ, Andersson M, Wiklund T, Joensuu T, van't Veer MB, Stovall M, Gospodarowicz M, Travis LB: Second malignant neoplasms among long-term survivors of Hodgkin's disease: a population-based evaluation over 25 years. J Clin Oncol 2002;20:3484-3494.

-5 Swerdlow AJ, Douglas AJ, Hudson GV, Hudson BV, Bennett MH, MacLennan KA: Risk of second primary cancers after Hodgkin's disease by type of treatment: analysis of 2,846 patients in the British National Lymphoma Investigation. BMJ 1992;304:1137-1143.

-6 Salas C, Pérez-Vera P, Frías S: Genetic abnormalities in leukemia secondary to treatment in patients with Hodgkin's disease. Rev Invest Clin 2011;63:53-63.

7 Várady E, Deák B, Molnár ZS, Rosta A, Schneider T, Esik 0, Eckhardt S: Second malignancies after treatment for Hodgkin's disease. Leuk Lymphoma 2001;42:1275-1281.

$\checkmark 8$ Maurizi Enrici R, Anselmo AP, Osti MF, Santoro M, Tombolini V, Mandelli F, Biagini C: Analysis of the risk of solid tumor following Hodgkin's disease. Haematologica 1997;82:57-63.

-9 Bonadonna G, Viviani S, Bonfante V, Gianni AM, Valagussa P: Survival in Hodgkin's disease patients report of 25 years of experience at the Milan Cancer Institute. Eur J Cancer 2005;41:998-1006.

10 Valagussa P, Santoro A, Kenda R, Fossati Bellani F, Franchi F, Banfi A, Rilke F, Bonadonna G: Second malignancies in Hodgkin's disease: a complication of certain forms of treatment. Br Med J 1980;280:216-219.

11 Valagussa P, Santoro A, Fossati Bellani F, Franchi F, Banfi A, Bonadonna G: Absence of treatment-induced second neoplasms after ABVD in Hodgkin's disease. Blood 1982;59:488-494.

12 Ellis M, Lishner M: Second malignancies following treatment in non-Hodgkin's lymphoma. Leuk Lymphoma 1993;9:337-342.

13 Travis LB, Curtis RE, Glimelius B, Holowaty EJ, Van Leeuwen FE, Lynch CF, Hagenbeek A, Stovall M, Banks PM, Adami J, Gospodarowicz MK, Wacholder S, Inskip PD, Tucker MA, Boice JD: Bladder and kidney cancer following cyclophosphamide therapy for non-Hodgkin's lymphoma. J Natl Cancer Inst 1995;87:524-530.

14 Bermejo JL, Sundquist J, Hemminki K: Bladder cancer in cancer patients: population-based estimates from a large Swedish study. Br J Cancer 2009;101:1091-1099. 